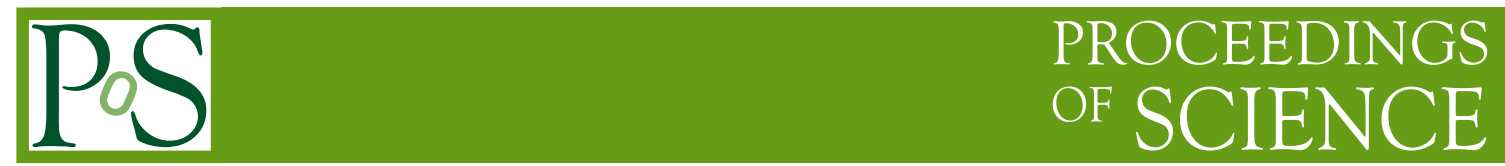

\title{
Searches for new physics with leptons and/or jets at CMS
}

\section{Carl VUOSALO**}

(The Ohio State University (US))

E-mail: cvuosalo@cern.ch

This talk will cover a wide range of new physics searches including many from the full dataset of $8 \mathrm{TeV}$ pp collision from 2012. Included in the talk will be new physics searches in decays to multi-jet final states, leptons plus jets, and also searches for heavy long-lived charged particles.

XXI International Workshop on Deep-Inelastic Scattering and Related Subjects 22-26 April, 2013

Marseilles, France

* Speaker.

${ }^{\dagger}$ On behalf of the CMS Collaboration 


\section{Introduction}

A major goal of the Compact Muon Solenoid (CMS) experiment [1] at the Large Hadron Collider is the discovery of new physics (NP) beyond the standard model (SM). Many NP theoretical models predict production of resonances that decay to paired jets or to leptons and jets. During 2012, the CMS detector recorded $19.6 \mathrm{fb}^{-1}$ of pp data at a center-of-mass energy of $8 \mathrm{TeV}$. Several CMS analyses exploited this rich data set to search for these NP signals.

\section{Search for a Heavy Neutrino and $W_{R}$}

A proposed, new right-handed $\mathrm{SU}_{\mathrm{R}}(2)$ symmetry group would produce new gauge bosons, called $\mathrm{W}_{\mathrm{R}}$ and $\mathrm{Z}$, and three heavy, right-handed neutrinos. Production of a $\mathrm{W}_{\mathrm{R}}$ resonance would produce two leptons and two jets:

$$
W_{R} \rightarrow \ell_{1} N_{\ell} \rightarrow \ell_{1} \ell_{2} W_{R}^{*} \rightarrow \ell_{1} \ell_{2} q q^{\prime} \rightarrow \ell_{1} \ell_{2} j j
$$

CMS has performed a search for this decay with $3.6 \mathrm{fb}^{-1}$ of $20128 \mathrm{TeV}$ pp data [2]. This search utilizes the $M_{\ell \ell j j}$ invariant mass distribution, in which the $\mathrm{W}_{\mathrm{R}}$ should produce a clear signal peak compared to the background, as shown in Fig. 1. Both electron and muon channels are included in the search. Background estimates were derived with data-driven techniques, except for the Z-plus-jets contribution, which was determined from simulation.
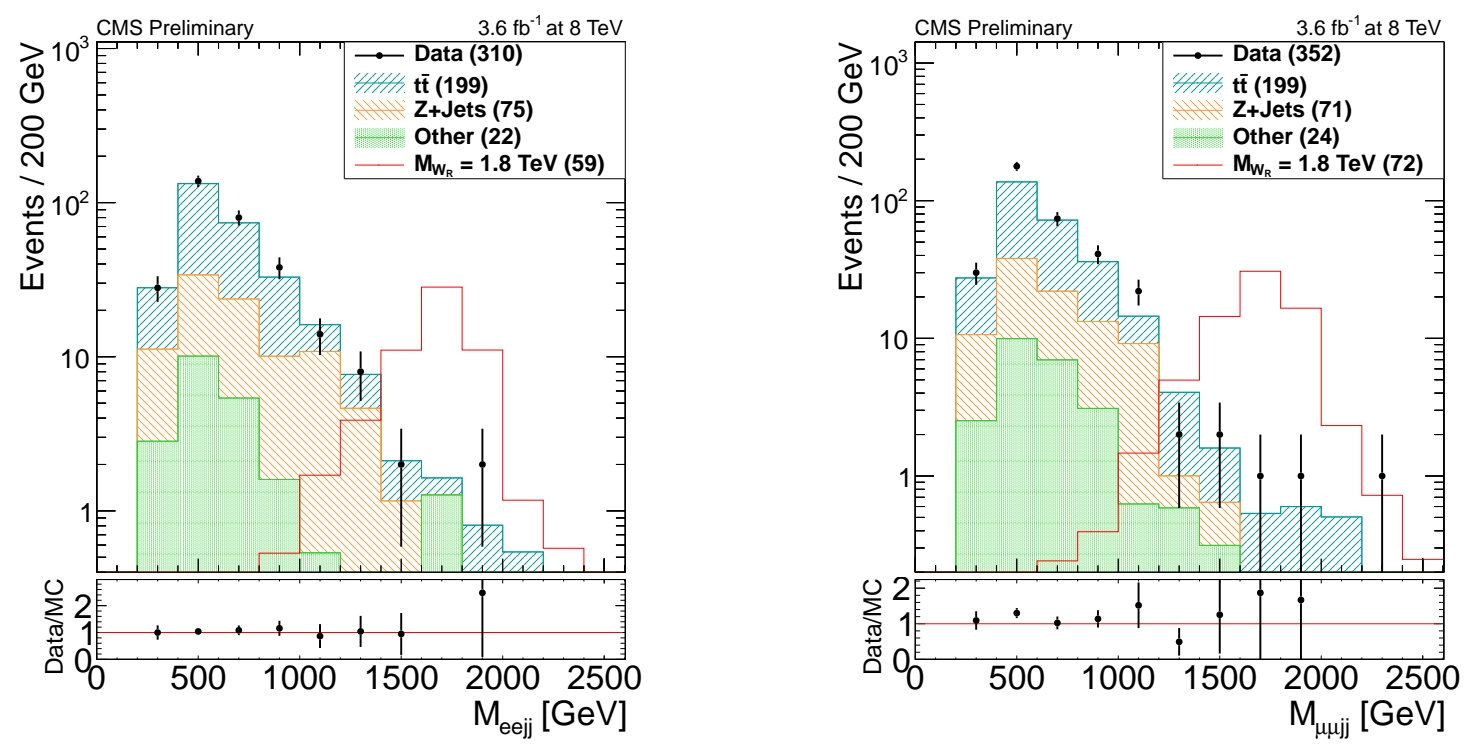

Figure 1: Four-object mass distribution for $e e j j$ (left) and $\mu \mu j j$ (right) events surviving event selection criteria using the collision data collected in 2012. The multijet and other minor SM backgrounds (e.g. diboson) are combined into a single "Other" category, and the signal mass point $M_{W_{R}}=1800 \mathrm{GeV}, M_{N_{\ell}}=$ $900 \mathrm{GeV}$, is included for comparison. The uncertainty in the data/MC ratio includes the statistical uncertainty on both the reconstructed events in data as well as the background expectation.

The systematic uncertainty for the signal is $15 \%$ and comes mostly from parton distribution function (PDF) uncertainty when generating the simulated signal samples. The background systematic uncertainties ranges from $20-50 \%$, which are mostly shape uncertainties. 
As can be seen in Fig. 1, data matches the background estimates within the uncertainties, and no significant excess is observed. Exclusion limits were calculated from a shape analysis of the $M_{\ell \ell j j}$ distribution. The best limits are achieved by combining the electron and muon channels, with the assumption of left-right symmetry $\left(g_{L}=g_{R}\right)$ and three degenerate generations of heavy neutrinos. These limits, as shown on the left in Fig. 2, extend up to $2800 \mathrm{GeV}$ on the mass of the $\mathrm{W}_{\mathrm{R}}$.
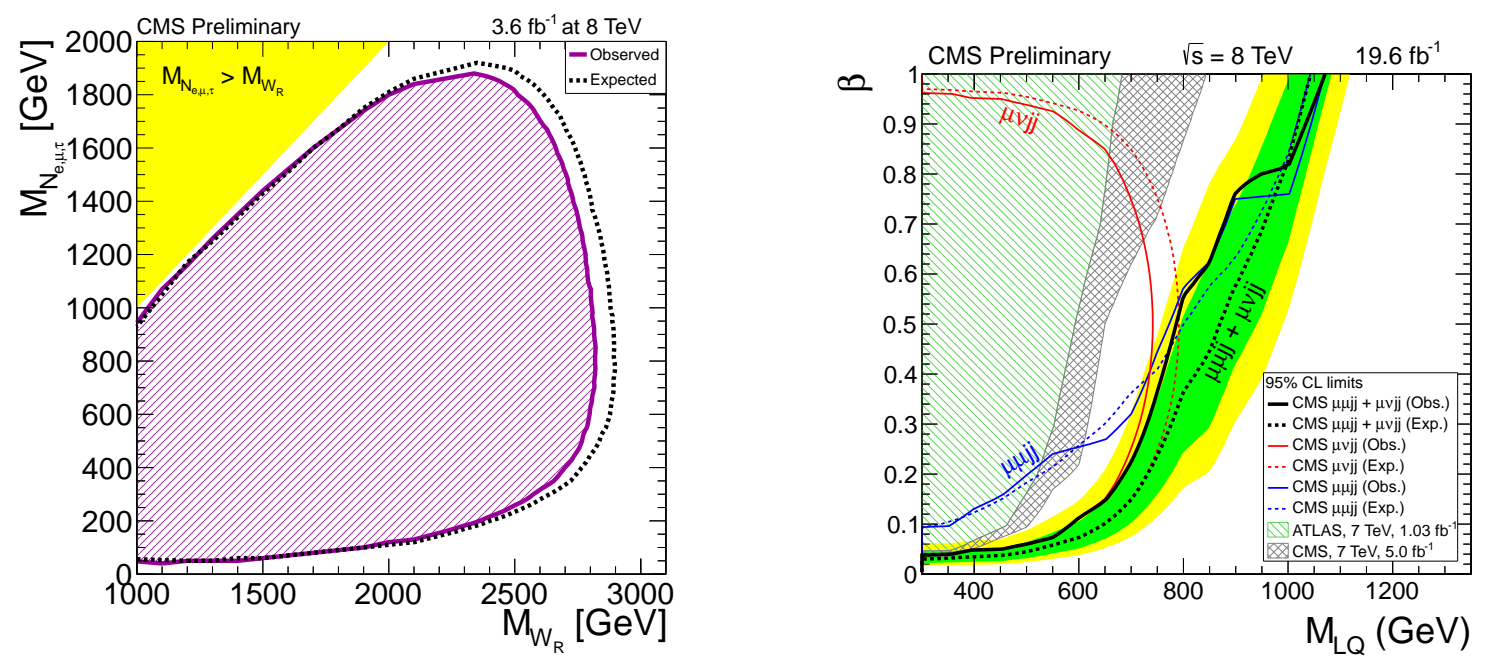

Figure 2: On the left, for the $\mathrm{W}_{\mathrm{R}}$ search, the $95 \%$ confidence level exclusion region in the $\left(M_{W_{R}}, M_{N_{\ell}}\right)$ plane obtained from combining the electron and muon channels. On the right, for the leptoquark search, the expected and observed exclusion limits at $95 \% \mathrm{CL}$ on second-generation leptoquark mass as a function of the branching fraction $\beta$. The dark green and light yellow expected limit uncertainty bands represent the $68 \%$ and $95 \%$ confidence intervals on the combination. Limits for the individual $\mu \mu j j$ and $\mu \nu j j$ channels are also given as in the $M_{\mathrm{LQ}}$ versus $\beta$ plane. Solid lines represent the observed limits in each channel, and dashed lines represent the expected limits. The leftmost shaded region is excluded by the most recent ATLAS 7TeV result, and the rightmost shaded region is excluded by the CMS 7 TeV Result.

\section{Search for Pair Production of Second-Generation Scalar Leptoquarks}

Leptoquarks are proposed particles with fractional charge that couple to leptons and quarks, have color, and can be scalar or vector particles. CMS has performed a search for pair production of scalar leptoquarks with the full $19.6 \mathrm{fb}^{-1}$ of $20128 \mathrm{TeV}$ pp data [3]. The search uses two signatures for leptoquark decay: a pair of leptoquarks each decaying to a muon and a jet (two muons and two jets); and one leptoquark decaying to a muon and a jet, and the second decaying to a neutrino and a jet (one muon, two jets, and missing energy).

The major backgrounds are from $\mathrm{W}$ plus jets and $\mathrm{t} \overline{\mathrm{t}}$ plus jets, and background estimates were obtained through data-driven techniques. The systematic uncertainty for signal is $5 \%$, mostly due to the uncertainty on the integrated luminosity measurement. The uncertainties for backgrounds range from 14-24\% and are mostly from jet energy resolution and muon energy scale uncertainties.

The number of observed data events matches the number of estimated background events within the uncertainties, so limits were calculated. Combining the limits for the two channels, and 
plotting them in terms of the leptoquark mass and the branching ratio of the leptoquark decaying to a lepton and quark, gives the results shown in Fig. 2. These limits extend up to $1070 \mathrm{GeV}$ on the leptoquark mass.

\section{Search for Long-lived Charged Particles}

Many extensions to the SM predict particles with mass greater than $100 \mathrm{GeV}$ and particles with fractional charge or with charge greater than the unit charge. Such particles could show very high or low energy loss as they travel through the detector (see the left plot of Fig. 4), have a long time of flight, or could switch between charged and neutral states while going through the detector. CMS has performed a search [4] for these particles with the combined 2011 and 2012 pp data at 7 and $8 \mathrm{TeV}$, which totals $23.8 \mathrm{fb}^{-1}$. The search was done in five channels: using only the CMS inner silicon detectors (tracker), using the tracker plus the muon system, using only the muon system, using the tracker to search for fractionally charged particles, and then using both systems to search for particles with charge greater than the unit charge. The background estimates for these searches were derived from data-driven methods. The systematic uncertainties on the signal acceptance range from $13-32 \%$, while the uncertainty on the dominant background is about $20 \%$. The data is found to match the background estimate within the uncertainties. Mass limits calculated at the 95\% confidence level (CL), shown in Fig. 3, are the most stringent to date for the signal models considered. The limits range up to $1322 \mathrm{GeV}$ on the gluino mass.
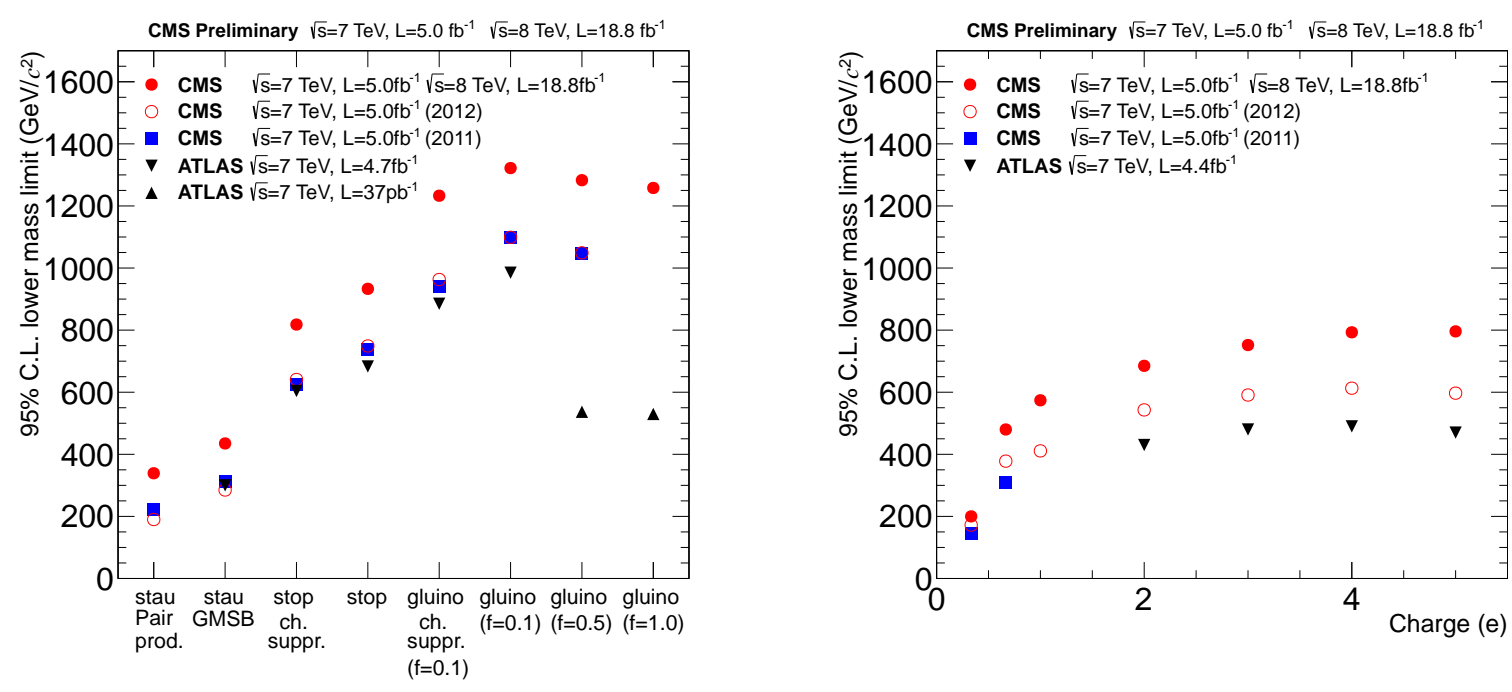

Figure 3: Mass lower limits at $95 \% \mathrm{CL}$ on various models compared with previously published results. Left: The model type is defined by the $x$ axis. Right: Mass limits versus electric charge.

\section{Search for Narrow Resonances using the Dijet Mass Spectrum}

Many new physics models predict heavy resonances that couple to quarks and gluons and decay to dijets. Some of these models include axigluons, color-octet colorons, excited quarks, 
Randall-Sundrum gravitons, scalar diquarks, string resonances, technicolor s8 resonances, W', and Z'. CMS has performed a search for such resonances with the full $19.6 \mathrm{fb}^{-1} 20128 \mathrm{TeV} \mathrm{pp}$ dataset [5]. This search employs the wide-jet technique which adds close sub-leading jets to the two leading jets in each selected event. The background prediction comes from a four-parameter fit to the data. The largest systematic uncertainty is the jet energy resolution uncertainty, which is $10 \%$. The data matches the background estimate with no excess or bumps observed on the smooth background. Mass limits are set on the eight signal models, as shown on the right in Fig. 4, with the strongest limit being $5.1 \mathrm{TeV}$ on the string resonance mass.
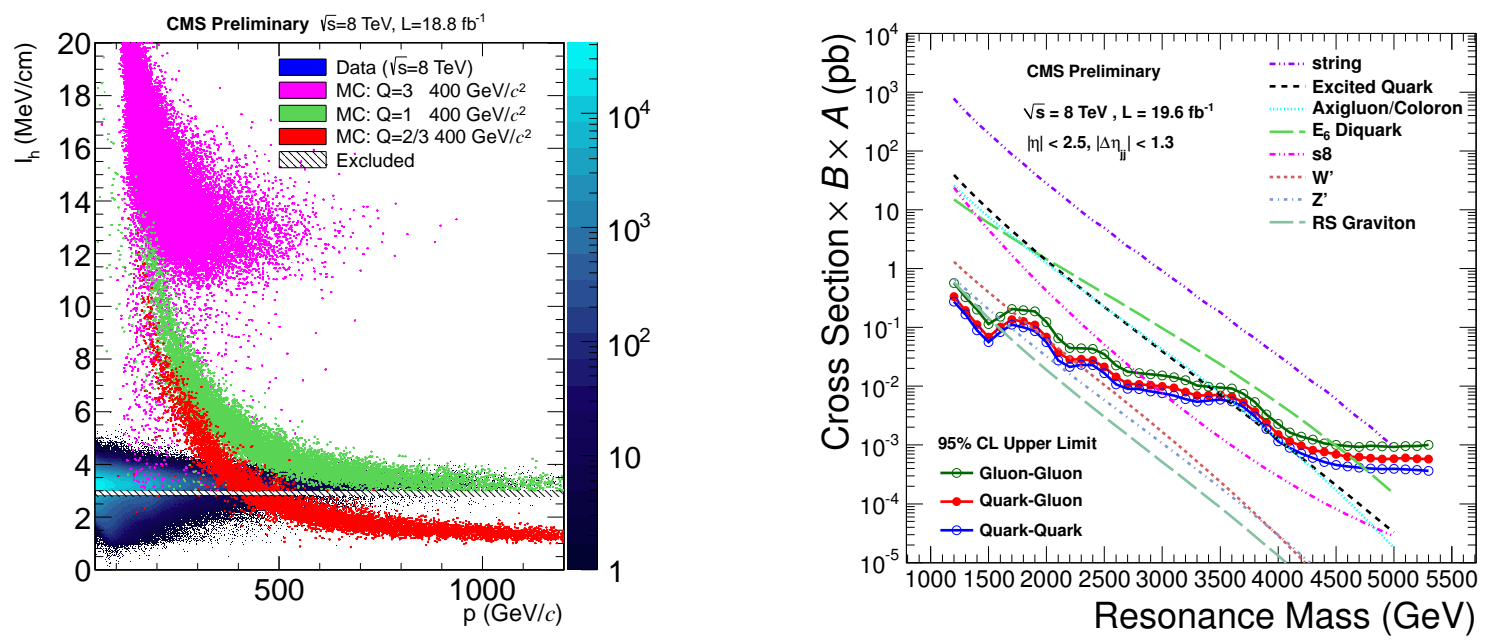

Figure 4: On the right, for heavy stable charge particle candidates, the distribution of $I_{h}$, which is a measure of particle energy loss over distance, versus particle momentum for data and singly, fractionally, and multiply charged candidates. On the left, for the dijet search, the observed 95\% CL upper limits on $\sigma \times B \times A$ for dijet resonances of the type gluon-gluon, quark-gluon, and quark-quark, compared to theoretical predictions for string resonances, $\mathrm{E}_{6}$ diquarks, excited quarks, axigluons, colorons, $\mathrm{s} 8$ resonances, new gauge bosons $\mathrm{W}^{\prime}$ and $\mathrm{Z}^{\prime}$, and RS gravitons.

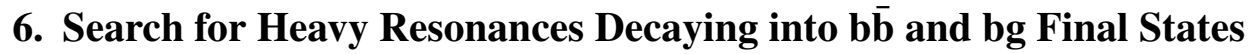

As a variation on the dijet search in Sec. 5, a b-jet tagging requirement can be placed on the jets in order to reduce SM backgrounds and to make the search sensitive to models that specifically produce b jets: excited b quarks, RS gravitons, and a sequential SM Z'. CMS has performed such a search with the full $19.6 \mathrm{fb}^{-1} 20128 \mathrm{TeV}$ pp dataset [6]. Like the previous analysis, this one uses the wide-jet technique and a background estimate from a four-parameter fit to the data. The jet energy resolution uncertainty of $10 \%$ is the largest systematic uncertainty. The data matches the background estimate, and no excess is observed. The best mass limits to date are set on the three signal models, as shown in Fig. 5, with the strongest limit being $1.7 \mathrm{TeV}$ on the $\mathrm{Z}$ ' mass.

\section{Conclusion}

The CMS Collaboration has performed several searches for new physics using leptons and jets 

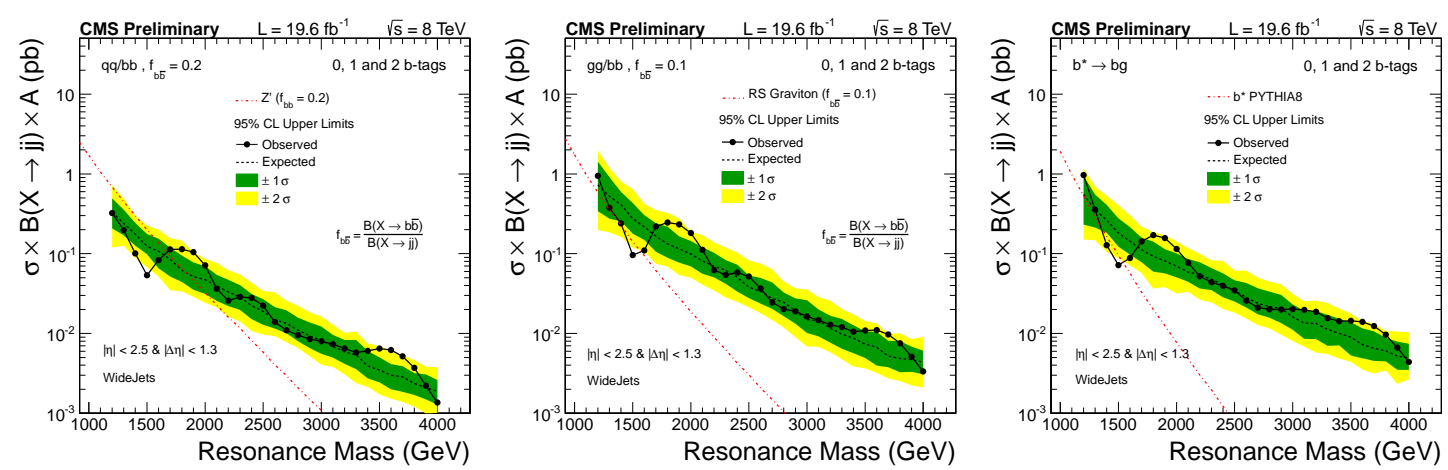

Figure 5: Combined observed and expected 95\% CL upper limits on $\sigma \times B \times A$ with systematic uncertainties included for qq/bb resonances with $f_{\mathrm{bb}}=0.2$ (left), gg/bb resonances with $f_{\mathrm{bb}}=0.1$ (middle), and $\mathrm{b}^{*} \rightarrow$ bg resonances (right). $f_{\mathrm{b} \bar{b}}$ is the ratio of the branching fraction of the resonance decaying to $b \bar{b}$ over the resonance decaying to all jets. Theoretical cross sections for RS graviton, Z', and excited b quark are shown for comparison.

with the $20128 \mathrm{TeV}$ pp collision dataset. No significant deviations from the SM were observed. New limits were set on many models, most being the best to date on these models. These limits range up to $2.8 \mathrm{TeV}$ on the mass of a $\mathrm{W}_{\mathrm{R}}$, up to $1.1 \mathrm{TeV}$ on the mass of scalar leptoquarks, up to $1.3 \mathrm{TeV}$ on high-mass supersymmetric particles with anomalous charges, up to $5.1 \mathrm{TeV}$ on dijet string resonances, and up to $1.7 \mathrm{TeV}$ on a $\mathrm{Z}$ '. CMS is continuing its searches for new physics, and more results will be coming out soon.

\section{References}

[1] CMS Collaboration, The CMS experiment at the CERN LHC, JINST 3 (2008) S08004, doi:10.1088/1748-0221/3/08/S08004.

[2] CMS Collaboration, Search for a heavy neutrino and right-handed $W$ of the left-right symmetric model in pp collisions at sqrt( $(s)=8 \mathrm{TeV}$, CMS Physics Analysis Summary CMS PAS EXO-12-017 (2012).

[3] CMS Collaboration, Search for pair production of second-generation scalar leptoquarks in pp collisions at sqrt(s) $=8 \mathrm{TeV}$ with the CMS detector, CMS Physics Analysis Summary CMS PAS EXO-12-042 (2012).

[4] CMS Collaboration, Search for Long-lived Charged Particles in pp collisions at sqrt( $s)=7$ and $8 \mathrm{TeV}$, CMS Physics Analysis Summary CMS PAS EXO-12-026 (2013).

[5] CMS Collaboration, Search for Narrow Resonances using the Dijet Mass Spectrum with $19.6 \mathrm{fb}^{-1}$ of $p p$ Collisions at sqrt $(s)=8 \mathrm{TeV}$, CMS Physics Analysis Summary CMS PAS EXO-12-059 (2013).

[6] CMS Collaboration, Search for Heavy Resonances Decaying into bbbar and bg Final States in pp Collisions at sqrt(s) $=8 \mathrm{TeV}$, CMS Physics Analysis Summary CMS PAS EXO-12-023 (2013). 